\title{
Plasma-stimulated pseudopod formation is increased in patients with elevated blood pressure
}

\author{
Hypertension Research (2011) 34, 787-789; doi:10.1038/hr.2011.30; published online 17 March 2011
}

There are numerous mechanisms that elevate blood pressure (BP) in hypertension, including the well-recognized roles of vasoconstriction and artery/arteriole restructuring. There is also an evidence for the involvement of a capillary mechanism, by which leukocytes, because of their greater size and rigidity, travel through the capillaries slower than erythrocytes, causing an accumulation of erythrocytes and resulting in a steep increase in the apparent viscosity. ${ }^{1}$ These effects in the microcirculation have a profound effect on capillary resistance and therefore on the BP upstream in the arterioles. ${ }^{1,2}$ Importantly, it is dependent on interaction between leukocytes and erythrocytes, but independent of leukocyte adhesion to the endothelium. ${ }^{3}$ This local increase in resistance is exacerbated, if leukocytes in the free circulation form pseudopods (PP), and therefore enlarge their surface area. It has been shown that whole blood from spontaneously hypertensive rats requires higher pressure than control animals to achieve the same flow rate, a finding attributed to the elevated number of circulating leukocytes with PP.,

Examination of PP formation in human hypertension has received little attention; however, non-invasive investigation of $\mathrm{PP}$ formation is possible through plasma stimulation of naïve cells. In this study, we examined associations between naïve neutrophil activation and BP. We hypothesized that PP formation would be greater in individuals with elevated BP than normal BP. Further, we examined the effect of a lifestyle intervention designed to increase cardiovascular fitness and reduce BP on PP formation capacity.

A total of 32 healthy, sedentary volunteers with normal or elevated BP (age 26-60 years; 19 female) gave written informed consent. The protocol was approved by the University of California, San Diego Institutional Review Board.
Cardiovascular fitness was assessed by maximal exercise test $\left(\mathrm{VO}_{2 \text { peak }}\right)$. Participant's $\mathrm{BP}$ was averaged from three seated, resting recordings. Subjects were grouped according to BP; Normal $\mathrm{BP}<120 / 80 \mathrm{~mm} \mathrm{Hg}(N=11,6$ female); Elevated $\mathrm{BP}>120 / 80 \mathrm{~mm} \mathrm{Hg} \quad(N=$ 21, 13 female). Heparinized plasma was harvested from whole blood drawn after a 20-min resting baseline.

The same young, asymptomatic donor provided naïve cells for all assays. Neutrophils were harvested from whole blood, isolated by two-step Histopaque-Percoll gradient centrifugation and resuspended in PBS $\left(2.4 \times 10^{7}\right.$ cells $/ \mathrm{ml}$ ). Donor neutrophils were incubated with participant plasma for $10 \mathrm{~min}$. Negative (PBS) and positive ( $N$-formyl-met-leu-phe (fMLP, Sigma-Aldrich, St Louis, MO, USA)) controls were run with each batch of samples. The reaction was fixed with glutaraldehyde (Sigma-Aldrich). Pictures of the first 100 cells were taken at $40 \times$, and analyzed using ImageJ (NIH, Bethesda, MD, USA) for PP formation and length. Any cell with a PP projection longer than 1 micron was considered activated (Figure 1 inset). PP length was defined as the difference between the average maximum diameters of the first five unactivated cells and the first 40 activated cells.

Elevated BP subjects $(N=21,13$ female) participated in a 12-week lifestyle intervention, after which $\mathrm{VO}_{2 \text { peak }}$ testing, BP measurement and blood sampling was repeated. Participants met with a personal trainer twice a week at a local YMCA and were encouraged to exercise an additional three times a week. In all, 11 participants also received diet advice from a registered dietician based on the 'Dietary Approaches to Stop Hypertension' (DASH). Given the small sample size and no statistical differences in intervention effects between groups, here, diet and nondiet participants were grouped together.
Participants in the elevated BP group were not different from those in the normal BP group in regard to $\mathrm{BMI}$, gender or $\mathrm{VO}_{2 \text { Peak, }}$ but were slightly older and as expected had higher resting SBP and DBP (Table 1).

Donor neutrophil PP length was significantly greater, when stimulated by plasma from elevated BP compared with normal $\mathrm{BP}$ subjects (mean \pm s.d. length $=2.48 \pm 0.42$ microns vs. $2.12 \pm 0.54$ microns, $P<0.05$ ). $\mathrm{PP}$ length positively correlated with subjects resting SBP $(r=0.415, \quad P=0.02)$ and resting DBP $(r=0.402, \quad P=0.02) \quad$ (Figure 1$)$, but was not significantly associated with BMI, age or $\mathrm{VO}_{2 \text { peak }}$.

As expected, lifestyle intervention significantly decreased resting SBP (mean \pm s.d. pre $=143.0 \pm 10.8 \mathrm{~mm} \mathrm{Hg}$, post $=135.4 \pm 10.1$ $\mathrm{mmHg}, \quad P<0.01$ ) and DBP (mean \pm s.d. pre $=86.8 \pm 7.0 \mathrm{~mm} \mathrm{Hg}$, post $=79.7 \pm 8.7 \mathrm{~mm} \mathrm{Hg}$, $P<0.001)$, and $\mathrm{VO}_{2 \text { peak }}$ showed a trend toward increase (mean \pm s.d. pre $=26.8 \pm 6.2 \mathrm{ml}$ $\mathrm{kg}^{-1} \mathrm{~min}^{-1}, \quad$ post $=28.8 \pm 6.9 \mathrm{ml} \mathrm{kg}^{-1} \mathrm{~min}^{-1}$, $P<0.06)$. A hierarchical linear regression was conducted using post-training PP length as the outcome variable, baseline PP length and age (Step 1), and change in resting SBP or DBP (Step 2) entered as predictor variables. A significant association emerged between change in DBP and post-training PP length $(\beta=-0.579$, $\left.\mathrm{B}=-0.140, r^{2}=0.227, P<0.05\right)$, but not with SBP $\left(\beta=-0.024, \mathrm{~B}=-0.005, r^{2}=0.000, P>0.5\right)$.

The current findings present the first evidence that PP formation might be enhanced in human hypertension: significantly longer pseudopods were projected by naïve cells stimulated by plasma from elevated BP compared with normal BP individuals. This association was confirmed by the observed continuous association between BP and PP length.

Consequences for increased PP length in the microcirculation are significant. ${ }^{2}$ 

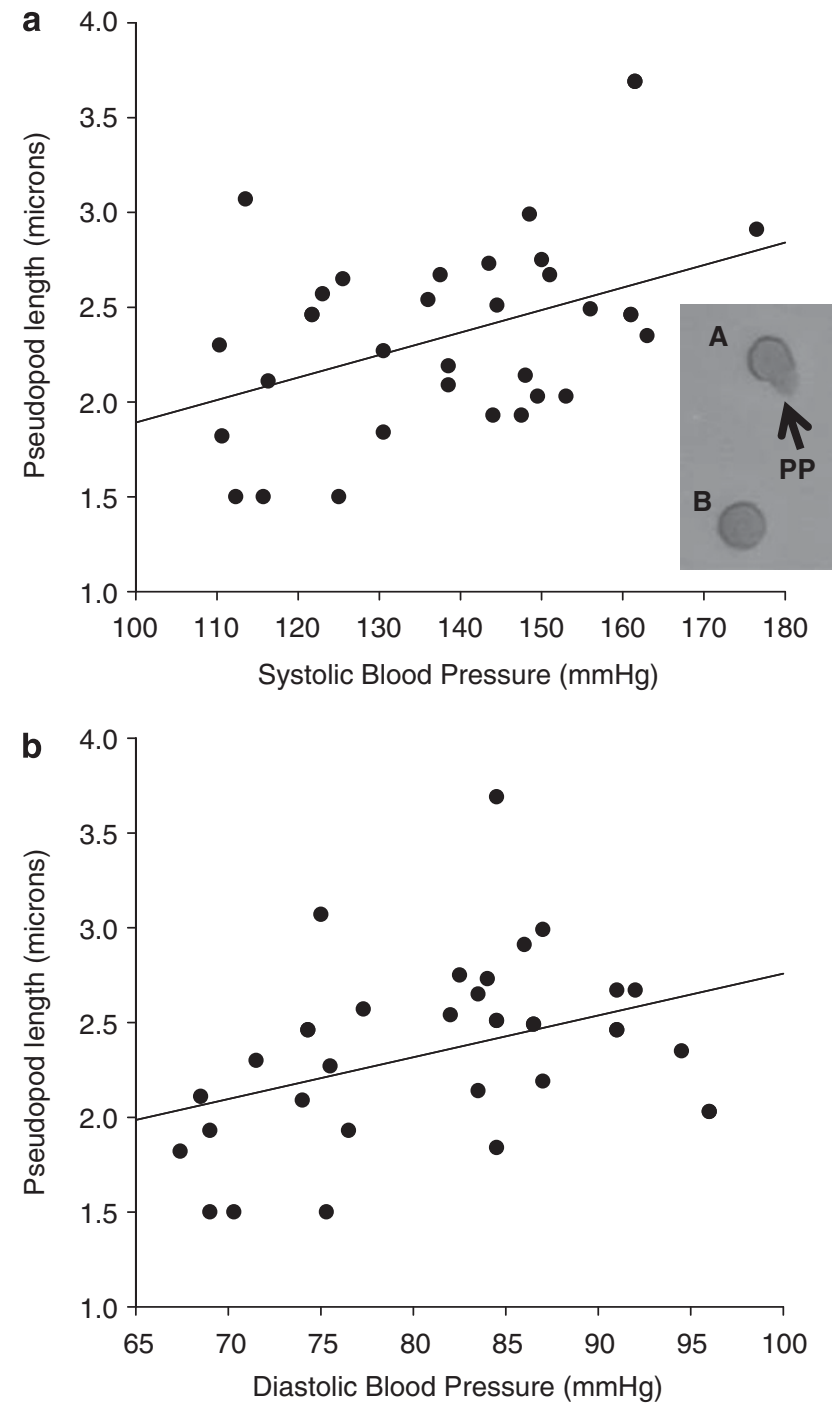

Figure 1 Pseudopod (PP) length vs. systolic blood pressure (SBP) (a; $r=0.415, P=0.018$ ) and diastolic blood pressure (DBP) (b: $r=0.402, P=0.023$ ). Inset, $P P$ morphology examples from cells stimulated with subject plasma, PP identified by arrows on cell $A$, cell $B$ showing no activation.

Table 1 Mean \pm s.d. participant characteristics

\begin{tabular}{lccr}
\hline & Elevated $B P(\mathrm{~N}=21)$ & Normal $B P(\mathrm{~N}=11)$ & \\
\hline Age (years) & $49.3 \pm 8.2$ & $41.2 \pm 8.7$ & $0.01^{\mathrm{a}}$ \\
Gender & $8 \mathrm{M}, 13 \mathrm{~F}$ & $5 \mathrm{M}, 6 \mathrm{~F}$ & 0.61 \\
$\mathrm{BMI}\left(\mathrm{kg} \mathrm{m}^{-2}\right)$ & $29.0 \pm 3.3$ & $29.8 \pm 3.6$ & 0.55 \\
VO & $2-$ Peak $\left(\mathrm{ml} \mathrm{kg}^{-1} \mathrm{~min}^{-1}\right)$ & $28.7 \pm 8.9$ & 0.97 \\
SBP $(\mathrm{mm} \mathrm{Hg})$ & $144.4 \pm 9.8$ & $119.9 \pm 10.1$ & $<0.001^{\mathrm{a}}$ \\
DBP $(\mathrm{mm} \mathrm{Hg})$ & $86.9 \pm 7.2$ & $76.3 \pm 7.8$ & $0.001^{\mathrm{a}}$ \\
\hline
\end{tabular}

Abbreviations: BP, blood pressure; BMI, body mass index; DBP, diastolic blood pressure; SBP, systolic blood pressure. a

Increased diameter of leukocytes will result in increased microvascular resistance, raising BP. $^{3}$ There are several ways through which PP formation is stimulated; the presence of a pro-inflammatory mediator or the absence/ inhibition of an anti-inflammatory mediator.
Alternatively, proteolytic activity may result in PPs by way of cleaving receptors, degrading mediators or releasing inflammatory epitopes. ${ }^{6}$ Determination of critical agonist components of blood might consider a range of possible targets, including: $\mathrm{N}$-formyl- methionyl-leucyl-phenylalanine (fMLP), platelet-activating factor, leukotriene B4, C5a anaphylotoxin and interleukin-8, which are known to induce PP projection. ${ }^{7,8}$

We also show preliminary data that $\mathrm{BP}$ reduction through lifestyle modification is related to reduction in PP stimulation. The direction of causality between reduction in BP and PP stimulation cannot be determined by the current analysis. It is interesting to note, however, that a significant association was observed between change in DBP after intervention, but not SBP, and although systolic BP is related to the contraction of the heart, diastolic BP is more closely related to the steady-state perfusion pressure of the vasculature, and thus the peripheral vascular resistance.

In summary, plasma from individuals with elevated BP stimulates greater PP formation than normal BP plasma. Further, BP reduction through lifestyle intervention is related to reduction of PP formation capacity. Given the potential for PP formation as a mediator of microvascular pressure, their role in human hypertension deserves further attention.

\section{CONFLICT OF INTEREST}

The authors declare no conflict of interest.

\section{ACKNOWLEDGEMENTS}

This work was supported by grant HL57265 and HL073355 from the National Institutes of Health and the UCSD General Clinical Research Center (MO1RR-00827).

Bassem M Shoucri ${ }^{1}$, Kate M Edwards ${ }^{2}$, Geert W Schmid-Schönbein ${ }^{1}$ and Paul J Mills ${ }^{2}$

${ }^{1}$ Department of Bioengineering, Whitaker Institute of Biomedical Engineering, University of California, San Diego, La Jolla, CA, USA and ${ }^{2}$ Department of Psychiatry, UCSD Medical Center, University of California, San Diego, La Jolla, CA, USA E-mail: kmedwards@ucsd.edu

1 Helmke BP, Bremner SN, Zweifach BW, Skalak R, Schmid-Schonbein GW. Mechanisms for increased blood flow resistance due to leukocytes. Am J Physiol. 1997; 273: H2884-H2890.

2 Fukuda S, Yasu T, Predescu DN, Schmid-Schonbein GW. Mechanisms for regulation of fluid shear stress response in circulating leukocytes. Circ Res 2000; 86: E13-E18. 3 Helmke BP, Sugihara-Seki M, Skalak R, Schmid-Schonbein GW. A mechanism for erythrocyte-mediated elevation of apparent viscosity by leukocytes in vivo without adhesion to the endothelium. Biorheology 1998; 35: 437-448. 
4 Shen K, Sung KL, Whittemore DE, DeLano FA, Zweifach BW, Schmid-Schonbein GW. Properties of circulating leukocytes in spontaneously hypertensive rats. Biochem Cell Biol 1995; 73: 491-500.

5 Schmid-Schonbein GW, Seiffge D, DeLano FA, Shen K, Zweifach BW. Leukocyte counts and activation in spon- taneously hypertensive and normotensive rats. Hypertension 1991; 17: 323-330.

6 Waldo SW, Rosario HS, Penn AH, Schmid-Schonbein GW. Pancreatic digestive enzymes are potent generators

of mediators for leukocyte activation and mortality. Shock 2003; 20: 138-143.
7 Zhelev DV, Alteraifi AM, Chodniewicz D. Controlled pseudopod extension of human neutrophils stimulated with different chemoattractants. Biophys J 2004; 87: 688-695.

8 Schmid-Schonbein GW. Analysis of inflammation. Annu Rev Biomed Eng 2006; 8: 93-131. 